\title{
Validación de un cuestionario de transgresiones ético-médicas.
}

Validation of a questionnaire dealing with medical ethics transgressions.

\author{
VÉLIZ MÁRQUEZ José1
}

\section{SUMMARY}

The increase in accusations against doctors about medical ethics transgressions, motivated, since 1999, the design and rehearsal of a questionnaire. This questionnaire collected the opinions about the transgression frequency observed by doctors in other colleagues. The fifth version of this questionnaire (22 items) was successfully subjected to validity and reliability tests, and the result was a sixth version with six new items. Objective: To validate the sixth version of the Transgressions Questionnaire. Material and methods: The sixth version was rehearsed in $\mathbf{5 3 0}$ doctors of 22 regional councils of the Peruvian Medical Association. The validity of theoretical construct was evaluated by means of the correlation of Pearson and the concurrent validity, comparing the preference order of the percentages only with the answers "frequent" or "very frequent" from 530 doctors, with the percentage achieved in a previous work on accusations against doctors. Results: The validity indexes of all items, even item 3 and 4 invalidated in the $5 t^{\mathrm{a}}$ version, fluctuated between $0.94-0.99$, higher then the results in the $5^{\text {ta }}$ version, verifying the construct validity. The preference order of percentages of the transgressions observed versus those transgressions reported (concurrent validation), was similar. The Crombach' alfa was 0.95 . Conclusions: The sixth version possesses theoretical construct validity and a concurrent validity. Six types of transgressions were identified and the most frequent transgression was related with the patient. (Medical-patient relationship). The use of these results is recommended in the continuous medical education and in pre and postgraduate levels. (Rev Med Hered 2006;17:212-219).

KEYWORDS: Medical ethics, medical error, ethic-medical transgressions.

Médico psiquiatra. Docente de la Universidad Peruana Cayetano Heredia. Bioeticista. Presidente del Comité de Ética en Investigación del Instituto Especializado en Salud Mental Honorio Delgado Hideyo Noguchi” 
Validación de un cuestionario de transgresiones ético-médicas.

\section{RESUMEN}

El incremento de las denuncias por transgresiones, en contra de los médicos, motivó, desde 1999, la confección y ensayo de un cuestionario que recoge opiniones sobre la frecuencia de transgresiones que los médicos observan en sus colegas, cuya quinta versión (22 ítemes) fuera sometida, exitosamente, a pruebas de validez y confiabilidad, resultando una sexta con 06 ítemes nuevos. Objetivo: El objetivo del presente estudio fue validar la sexta versión del Cuestionario de Transgresiones. Material y métodos: La sexta versión se aplicó en 530 médicos de 22 consejos regionales del Colegio Médico. La validez de constructo teórico se evaluó por medio de la correlación r de Pearson y la concurrente comparando el orden de prelación de porcentajes de respuestas "frecuente" o "muy frecuente" de los 530 médicos, con los alcanzados en un trabajo anterior sobre denuncias en contra de los médicos. Se evaluó la confiabilidad por medio del alfa de Crombach. Resultados: Los índices de validez de todos los ítemes, incluso el 3 y el 4 invalidados en la Quinta versión, fluctuaron entre 0,94 y 0,99 comprobando la validez de constructo. La validación concurrente se estableció comparando el orden de prelación de los porcentajes de las transgresiones observadas y las denunciadas que resultó igual. El alfa de Crombach fue de 0,95. Conclusiones: La sexta versión posee validez de constructo teórico y concurrente así como confiabilidad. Se identificaron 6 tipos de transgresiones, siendo la más frecuentemente elegida la que está en relación directa con el paciente (relación médico-paciente). Se recomienda la utilización de estos hallazgos, en educación médica continua y en pre y postgrado.(Rev Med Hered 2006;17:212-219).

PALABRAS CLAVE: Ética médica, error médico, transgresiones ético-médicas.

\section{INTRODUCCIÓN}

Cuando en 1998 fui invitado a participar como miembro del Comité de Vigilancia Ética y Deontológica del Consejo Nacional (CVED-CN) del Colegio Médico, encontré que no se conocía trabajo alguno que resumiera los esfuerzos hechos por nuestros antecesores, y por lo tanto, cada comité, al ser elegido cada dos años, iniciaba sus labores casi desde cero, limitándose a discutir sobre las apelaciones generadas desde los consejos regionales y una que otra consulta de otras instituciones.

Si bien es cierto que también se realizaban esfuerzos por difundir conocimientos de ética médica y bioética, éstos eran propuestos más intuitivamente, que basados en un diagnóstico de la situación de la conducta moral de los médicos, a pesar de que en el mismo local funciona el Consejo Regional III de Lima, que es el que, por congregar a casi el 50\% de los médicos de todo el país, tiene en sus archivos valiosísimo material que estaba en espera de ser utilizado.

La delicada tarea de calificar la conducta moral de nuestros pares, requería también premunirse de aspectos teóricos, por lo que no dudé en participar en la primera diplomatura de ética médica en la Universidad Nacional Mayor de San Marcos, a la par que iniciaba una investigación en los expedientes del citado Consejo Regional sobre las denuncias en contra de los médicos (1).
La frecuencia de denuncias, podía tomarse como un esbozo de diagnóstico situacional moral del médico, pero sólo según opinión de los denunciantes. Aun así, resultó de lo más interesante, ya que las supuestas transgresiones denunciadas, establecían por primera vez, una taxonomía más variada que la esperada, puesto que al lado del 38\% de denuncias "por transgresiones en relación directa con pacientes”, se presentaba un $28 \%$ "por transgresiones a reglamentos o códigos de salud", un sorprendente $14 \%$ "por agresión verbal o física entre médicos", $11 \%$ "por transgresiones en el ámbito penal” y 9\% "por falsificación de certificado médico u otro documento". Estos datos sugirieron diversas hipótesis y las respectivas rutas de investigación. Una de ellas relacionada con la necesidad de comparar las opiniones de los denunciantes (más frecuentemente pacientes), con las de los médicos respecto a la frecuencia de transgresiones que ellos mismos observaban a su alrededor.

Así, pensé, se podía llegar a lo que, según mi parecer, aún constituye un problema fundamental: el estudio de los procesos que llevan al error médico y consecuentemente a la transgresión de las normas, en particular las contenidas en nuestro Código de Ética, ya que en el país se desconoce la frecuencia de las mismas.

Por otro lado, no es factible imitar diseños de investigación, en base al estudio de las historias clínicas, como el Harvard Medical Practice Study (2), por ser 
muy costosos. En cambio, era posible explorar las opiniones sobre las trasgresiones de normas que ellos cometen, o al caer eventualmente en situaciones tales como la de inculpado, culpable sentenciado o denunciado inocente, que son las situaciones estudiadas a través de los expedientes de denuncias, faltando las opiniones cuando actúan como simples observadores participantes, que es la que procedimos a investigar a través de un cuestionario que, aparte de brindarnos datos directos y actuales, podía sugerirnos contenidos y estrategias de educación médica continua en el campo de la ética médica.

Con anuencia del CVED-CN, se revisaron los planes operativos en educación continua ético médica que se llevaron a cabo desde 1998, incluyendo investigaciones científicas que, en parte, ya han sido publicadas (3) y otras referidas en las respectivas memorias que se elevan al Decano Nacional (4).

Se tomaron algunos itemes de un pequeño cuestionario de 17 transgresiones preparado el año 1997 por el Dr. Hector Tovar y, añadiendo otros se realizó un estudio piloto utilizando una primera versión de una encuesta anónima, para someterla a la opinión y crítica de los miembros del CVED-CN 1999-2000, y ulterior aplicación el 4 de setiembre de 1999, a 24 médicos participantes del cursillo "Problemas en la práctica médica”.

Una segunda versión fue respondida por 55 participantes del Seminario-taller "El nuevo Código y los comités de ética”, el 27 de abril del 2000, dando lugar a la tercera versión de 58 ítems, que fuera evaluada por los 14 miembros del CVED-CN 2000-2001, el 24 de octubre del 2000, en un intento fallido de validación, pero que generó valiosas sugerencias como el evitar las oraciones negativas, por ejemplo: "Estar desactualizado" en lugar de "No estar actualizado", "Insuficiente información al paciente" en lugar de "No informar al paciente"; o añadir: "sin que esté autorizado" a "Practicar aborto"; y un nuevo ítem: "Dar certificado o informe falso", con todo lo cual se generó la cuarta versión de 62 ítems distribuidos en 6 secciones.

En el 2002, en el contexto de la Maestría Internacional de Bioética, sometí el cuestionario a pruebas de validación y confiabilidad, pero, por ser un cuestionario demasiado amplio y considerar variados aspectos teóricos, se tomó sólo la primera sección A (Transgresiones de los médicos), a fin de presentarla ante la Comisión Revisora de la Universidad Nacional Mayor de San Marcos cuyas observaciones formales fueron incorporadas, convirtiéndose así en la quinta versión de 22 ítemes (5).

La validez de contenido según el criterio de los 17 miembros del CVED-CN y del CVED del Consejo Regional III Lima (CVED-CRIII) 2002-2003, del Colegio Médico, como jueces expertos, confirmaron que todos los ítemes propuestos correspondían a transgresiones ético-médicas.

La validez de constructo teórico se determinó en el retest de los mismos jueces, utilizando la correlación ítem-test con una $r$ de PEARSON que alcanzó valores entre 0,64 y 0,89 con la excepción del item 3 ( $\mathrm{r}=$ 0,18), siendo el mínimo tolerable, según Garret, de 0,20 (3). También se utilizaron las opiniones vertidas en el Cuestionario, de una muestra de 76 médicos de Ayacucho, Ica, Huaraz y Huánuco, en la que, la correlación ítem-test, encontró una r entre 0,40 y 0,84 excepto el ítem $4(r=0,13)$. Así pues, el ítem 3 en los jueces, y el 4 en la muestra de provincias, resultaron invalidados.

Como aproximación a la validez concurrente, se estableció comparación con la muestra de 148 expedientes de denuncias de anterior investigación científica (1), comprobándose que el orden de prelación de los porcentajes de las transgresiones denunciadas era igual al conseguido por el cuestionario respecto a las transgresiones "observadas". Así se concluyó que el cuestionario era válido en 20 de sus 22 ítems. La confiabilidad se estableció por medio de la correlación test-retest $(0,77, \mathrm{p}<0,001)$ y el alfa de Crombach (6).

En los siguientes seminario-talleres realizados, los medicos encuestados sugirieron seis nuevos itemes que ha permitido elaborar una sexta versión, cuya validación y confiabilidad presentamos en el presente estudio.

\section{MATERIAL Y MÉTODOS}

La sexta versión cuenta con 28 ítemes (Anexo $\mathrm{N}^{\circ} 1$ ), sin dejar de lado los dos que resultaron invalidados (los ítemes 3 y 4, de la quinta versión), por considerar que sus importantes contenidos merecían permanecer bajo evaluación, hasta decidir si se aceptaban o no en el Cuestionario. Ha sido aplicado durante los últimos 22 seminario-talleres, llevados a cabo por el CVED-CN del CMP entre mediados del 2004 y fines del 2005.

Los 6 nuevos ítemes incorporados fueron los siguientes:

Ítem 5.- Usan medidas extraordinarias en pacientes terminales (Distanasia).

Ítem 6.- Ayudan al paciente a ejecutar el acto suicida 
Validación de un cuestionario de transgresiones ético-médicas.

Anexo $N^{\circ} 1$. Cuestionario de transgresiones.

No escriba apellidos o nombres. Deseamos su colaboración para conocer la frecuencia que, según sus observaciones, tienen las transgresiones ético-médicas* que mencionamos. Los datos servirán al Colegio Médico para elegir los contenidos de futuros cursos de ética, mejorar otras actividades o emprender nuevas. Muy agradecidos.

Fecha...............Profesión: ............... Especialidad:

¿Tiene título de especialista otorgado por universidad? SI NO

Años de experiencia profesional.......... SEXO : Masc. Fem. EDAD.

¿Trabaja con pacientes? NO SI.... ¿Dónde?

¿Trabaja como docente NO SI.... ¿ Dónde ?

Señale la frecuencia de los transgresiones listadas, escribiendo en los cuadraditos de la derecha, el número respectivo, según la siguiente escala:
1. Nunca
2. Rara vez
3. A veces, promedio
4. Frecuente
5. Muy frecuente

A. ALGUNOS MÉDICOS......

1. Están desactualizados en sus conocimientos médicos

2. Están desactualizados en sus conocimientos en ética médica

3. Usan inadecuadamente o abusan de la publicidad

4. Practican eutanasia

5. Usan medidas extraordinarias en pacientes terminales (Distanasia)*

6. Ayudan al paciente a ejecutar el acto suicida (Suicidio asistido)

7. Practican el aborto sin que esté indicado

8. Explotan económicamente a sus pacientes*

9. Acosan sexualmente o mantienen relaciones sexuales con sus pacientes

10. Violan el secreto profesional

11. Tratan de modo descortés a sus pacientes

12. Tratan de modo discriminatorio a sus pacientes.

13. Influyen políticamente en sus pacientes.

14. Influyen en aspectos religiosos en sus pacientes.

15. Prescriben o realizan tratamientos ineficaces.

16. Prescriben o realizan tratamientos de modo imprudente * o audaz.

17. Prescriben o realizan tratamientos innecesarios (Ej.: cesárea).

18. Olvidan u omiten indicaciones perjudicando al paciente.

19. Tratan pacientes que no son de su especialidad.

20. Realizan la historia clínica de modo incompleto.

21. Registran mal los datos en la historia clínica (Ej.: ilegible).

22. Informan insuficientemente al paciente.

23. Venden muestras médicas o material médico a los pacientes.

24. Expiden certificados o informes falsos.

25. Emplean escaso tiempo para evaluar al paciente.

26. Exhiben comportamiento público antiético o inmoral.

27. Denigran, difaman o hablan mal de otros colegas.

28. Maltratan, abusan o explotan a colegas o personal de salud. ¿Otras transgresiones?, Señale cuáles y califíquelas

\section{Glosario}

\footnotetext{
-Trasgresión ético-médica: Conducta antiética que perjudica directa o indirectamente al paciente

-Distanasia o encarnizamiento terapéutico

-Ítem 8: incluye inducir a consulta particular, cobro indebido o excesivo, etc.

-Tratamiento ineficaz: No surte el efecto deseado. (Difiere del efecto placebo, que es a sabiendas).

-Tratamiento imprudente: Utilizado a pesar del alto riesgo para el paciente.
} 
(Suicidio asistido)

Ítem 25.- Emplean escaso tiempo para evaluar al paciente.

Ítem 26.- Exhiben comportamiento público antiético o inmoral.

Ítem 27.- Denigran, difaman o hablan mal de otros colegas.

Ítem 28.- Maltratan, abusan o explotan a colegas o personal de salud.

Observando el contenido de los 28 ítemes, se delimitaron las siguientes áreas o campos:

Área de transgresiones en relación directa con el paciente: Ítemes $8,11,12,13,14,22$, y 25, en los que el acto médico se realiza directamente con el paciente, y que incluye tratamientos referidos en los itemes 5, 15, 16, 17, 18 y 19, en los que, el acto médico con el paciente, incorpora la prescripción o no prescripción de un fármaco o intervención como resultado del proceso diagnóstico.

Área de la historia clínica: Ítemes 20 y 21, dada la gran importancia de este documento tanto para el paciente como para el médico, incluso en los aspectos legales.

Área de educación médica continua: Ítemes 1 y 2, que atañe a las transgresiones del deber de actualización permanente.

Área económica: Ítemes 3 , 8 y 23 en los que lo prioritario es el aspecto crematístico.

Área de connotación penal: Ítemes 4, 6, 7, 9, 10 y 24, en los que prepondera la tendencia a la comisión de un delito.

Área de relaciones sociales extra clinicas: Ítemes 26, 27 y 28, que se ocupa de las perturbaciones de las relaciones con otros grupos diferentes al de los pacientes.

No todas las áreas son excluyentes, así por ejemplo el ítem "Explotan económicamente a sus pacientes", podría ser considerado en el "Área de Trasgresiones en relación directa con el paciente", pero se coloca dentro del "Área económica" por ser opinión del medico y no del paciente.

A pesar de la escasez de financiamiento y de las dificultades del trabajo de campo, se pudo recolectar una muestra intencional de 530 sujetos, médicos interesados en el tema, y por lo tanto fructífera en cuanto a la validación de contenidos. Todos ellos participaron de los seminarios talleres sobre "Evaluación ética de las denuncias" o sobre "Aspectos éticos y legales del acto médico", especialmente preparados para los médicos miembros de los comités de ética y de asuntos contenciosos, de los consejos regionales de Arequipa, Ayacucho, Cusco, Chiclayo, Chimbote, Huancavelica, Huancayo, Huanuco, Huaraz. Ica, La Merced, LimaCallao, Lima-provincias, Moquegua, Pasco, Piura, Pucallpa, Puerto Maldonado, Puno, Tacna, Trujillo y Tumbes.

Se añadió al glosario una breve definición del término “distanasia” (Anexo $\mathrm{N}^{\circ} 1$ ) al no existir en la anterior versión. Se elaboraron cuadros de frecuencias y porcentajes, en particular las referidas a las elecciones "frecuente" y muy frecuente". La validez de constructo teórico, se avaluó con la correlación ítem-test. La validación concurrente, comparando los órdenes de prelación de los porcentajes de tipos de transgresiones denunciadas (1) con las “observadas” según opinión de los 530 médicos. La validez de contenido fue evaluada parcialmente al solicitarse que, si les parecía que alguno de los ítemes no correspondían a una transgresión ético-medica, lo anotaran en la parte final del formato. La confiabilidad fue evaluada por medio del alfa de Crombach.

\section{RESULTADOS}

Los índices de validez del constructo teórico de los 28 ítemes superaron el 0.20 , límite inferior recomendado por Garret (3), variando entre 0,93 y 0,99, incluyendo a los ítemes 3 y 4 no válidos en la versión anterior (Tabla No 1 ).

En la Tabla $\mathrm{N}^{\circ} 2$ se presenta la cantidad de elecciones, por ítem, referidas como frecuente o muy frecuentemente "observadas" con los respectivos porcentajes. Ocupa el primer lugar los actos médicos “en relación directa con pacientes" que incluye los tratamientos, alcanzando un $40 \%$ del total. El segundo lugar, con $17 \%$, lo ocupa las transgresiones en la confección de la historia clínica y con 15\% las que incumplen el deber de actualización permanente. Menores cantidades corresponden a las áreas "económica”, “sociales extreclínicas” y “de connotación penal” con porcentajes de 10,6; 8,7 y 8,5 respectivamente.

Estas transgresiones "observadas" frecuente o muy frecuentemente, por 530 médicos, las comparamos con las denunciadas referidas en el trabajo antes mencionado (1), resultando la Tabla $N^{\circ} 3$, en la que se obvia las áreas “observadas” “de la historia clínica” y de “educación médica continua”, por razones que expondremos en la Discusión. En esta Tabla 


\section{Tabla $\mathrm{N}^{\circ} 1$. Índices de validez de los 28 ítems de la sexta versión del cuestionario de transgresiones $(n=530$ sujetos).}

1. Están desactualizados en sus conocimientos médicos

2. Están desactualizados en sus conocimientos en ética médica

3. Usan inadecuadamente o abusan de la publicidad

4. Practican eutanasia

5. Usan medidas extraordinarias en pacientes terminales (Distanasia)

6. Ayudan al paciente a ejecutar el acto suicida (Suicidio asistido)

7. Practican el aborto sin que esté indicado

8. Explotan económicamente a sus pacientes

9. Acosan sexualmente o mantienen relaciones sexuales con sus pacientes

10. Violan el secreto profesional

11. Tratan de modo descortés a sus pacientes

12. Tratan de modo discriminatorio a sus pacientes.

13. Influyen políticamente en sus pacientes.

14. Influyen en aspectos religiosos en sus pacientes.

15. Prescriben o realizan tratamientos ineficaces.

16. Prescriben o realizan tratamientos de modo imprudente o audaz.

17. Prescriben o realizan tratamientos innecesarios (Ej.: cesárea).

18. Olvidan u omiten indicaciones perjudicando al paciente.

19. Tratan pacientes que no son de su especialidad.

20. Realizan la historia clínica de modo incompleto.

21. Registran mal los datos en la historia clínica (Ej.: ilegible).

22. Informan insuficientemente al paciente.

23. Venden muestras médicas o material médico a los pacientes.

24. Expiden certificados o informes falsos.

25. Emplean escaso tiempo para evaluar al paciente.

26. Exhiben comportamiento público antiético o inmoral.

27. Denigran, difaman o hablan mal de otros colegas.

28. Maltratan, abusan o explotan a colegas o personal de salud. 
Tabla $\mathrm{N}^{\circ} 2$. Porcentajes de elecciones "frecuente" o "muy frecuente" según área de transgresiones e ítemes. Lima y provincias ( $n=3501$ elecciones).

\begin{tabular}{|c|c|c|}
\hline$\underline{\text { Áreas e ítemes }}$ & Elecc. & $\%$ \\
\hline \multicolumn{3}{|l|}{ ÁREA RELACIÓN DIRECTA CON PACIENTE : } \\
\hline $\begin{array}{l}\text { 5. Usan medidas extraordinarias en pacientes terminales } \\
\text { 11. Tratan de modo descortés a sus pacientes }\end{array}$ & 176 & $\begin{array}{l}1,25 \\
5,02\end{array}$ \\
\hline 12. Tratan de modo discriminatorio a sus pacientes. & 145 & 4,14 \\
\hline 13. Influyen políticamente en sus pacientes & 36 & 1,02 \\
\hline 14. Influyen en aspectos religiosos en sus pacientes & 9 & 0,25 \\
\hline 15. Prescriben o realizan tratamientos ineficaces & 73 & 2,08 \\
\hline 16. Prescriben o realizan tratamientos de modo imprudente o audaz & 56 & 1,59 \\
\hline 17. Prescriben o realizan tratamientos innecesarios (Ej.: cesárea). & 134 & 3,82 \\
\hline 18. Olvidan u omiten indicaciones perjudicando al paciente & 67 & 1,91 \\
\hline 19. Tratan pacientes que no son de su especialidad. & 220 & 6,28 \\
\hline 22. Informan insuficientemente al paciente. & 197 & 7,11 \\
\hline 25. Emplean escaso tiempo para evaluar al paciente. & 202 & $\begin{array}{r}5,62 \\
(40 \%)\end{array}$ \\
\hline \multicolumn{3}{|l|}{ ÁREA HISTORIA CLÍNICA } \\
\hline 20. Realizan la historia clínica de modo incompleto. & 296 & 8,45 \\
\hline 21. Registran mal los datos en la historia clínica (Ej.: ilegible). & 295 & $\begin{array}{r}8,42 \\
(17 \%)\end{array}$ \\
\hline \multicolumn{3}{|l|}{ ÁREA EDUCACIÓN MÉDICA CONTINUA } \\
\hline 1. Están desactualizados en sus conocimientos médicos & 148 & 4,22 \\
\hline 2. Están desactualizados en sus conocimientos en ética médica & 378 & $\begin{array}{l}10,79 \\
(15 \%)\end{array}$ \\
\hline \multicolumn{3}{|l|}{ ÁREA ECONÓMICA } \\
\hline $\begin{array}{l}\text { 3. Usan inadecuadamente o abusan de la publicidad } \\
\text { 8. Explotan económicamente a sus pacientes }\end{array}$ & $\begin{array}{c}177 \\
154\end{array}$ & $\begin{array}{l}5,05 \\
4,39\end{array}$ \\
\hline 23. Venden muestras o material médico a sus pacientes & 43 & 1,22 \\
\hline \multicolumn{3}{|l|}{ ÁREA DE RELACIONES SOCIALES EXTRACLÍNICAS } \\
\hline 26. Exhiben comportamiento público antiético o inmoral & 48 & 1,37 \\
\hline 27. Denigran, difaman o hablan mal de otros colegas. & 160 & 4,57 \\
\hline 28. Maltratan, abusan o explotan a colegas o personal de salud & 97 & 2,77 \\
\hline \multicolumn{3}{|l|}{ ÁREA DE CONNOTACIÓN PENAL } \\
\hline $\begin{array}{l}\text { 4. Practican eutanasia } \\
\text { 6. Ayudan al paciente e ejecutar el acto suicida (suicidio asistido) }\end{array}$ & $\begin{array}{l}4 \\
1\end{array}$ & $\begin{array}{l}0,11 \\
0,02\end{array}$ \\
\hline 7. Practican el aborto sin que esté indicado & 126 & 3,59 \\
\hline 9. Acosan sexualmente o tienen relaciones sexuales con sus pacientes & 14 & 0,39 \\
\hline 10. Violan el secreto profesional & 73 & 2,08 \\
\hline 24. Expiden certificados o informes falsos & 81 & 2,31 \\
\hline
\end{tabular}

Tabla $N^{\circ} 3$. Tipos de transgresiones observadas versus denunciadas.

\begin{tabular}{lcccc}
\hline & \multicolumn{2}{c}{ Observadas } & \multicolumn{2}{c}{ Denunciadas } \\
\cline { 2 - 5 } & $\mathrm{n}$ & $(\%)$ & $\mathrm{n}$ & $(\%)$ \\
\hline Directa con pacientes & 1402 & $(40,0)$ & 55 & $(33,9)$ \\
Económica & 374 & $(10,6)$ & 33 & $(20,3)$ \\
Sociales extraclínicas & 305 & $(8,7)$ & 22 & $(13,5)$ \\
Connotación penal & 299 & $(8,5)$ & 21 & $(12,9)$ \\
No comparables & 1121 & $(32,0)$ & 31 & $(19,1)$ \\
& 3501 & $(99,8)$ & 162 & $(19,1)$ \\
\hline
\end{tabular}

constatamos que el orden de prelación (1ro al 4to) de las áreas de porcentajes comparables "observados", es igual en los denunciados.

En cuanto a la validez de contenido nadie opinó en contra de que alguno de los ítemes propuestos correspondiera a transgresiones ético-médicas observables.

La confiabilidad, evaluada con el alfa de Crombach, resultó en un puntaje de 0,95.

\section{DISCUSIÓN}

Ante la carencia del necesario financiamiento, no fue factible intentar una investigación como la realizada por Brennan et al (2), pero, considerando que los médicos tienen información directa y vivencial respecto a las transgresiones ético-médicas, es decir, según el lenguaje antropológico, son “observadores participantes", hemos recogido sus opiniones, a través de la sexta versión, variante de la quinta del Cuestionario de Transgresiones, anteriormente validado y sometido a pruebas de confiabilidad.

Como lo recomienda Garret, todo instrumento validado, debe proseguir con ulteriores validaciones para reafirmar su calidad, que es precisamente lo que hemos realizado con la sexta versión del cuestionario, resultando los altos índices mostrados de la tabla $\mathrm{N}^{\circ} 2$ que comprueban la validez de constructo teórico, superior a la de la quinta versión.

La comparación entre las transgresiones observadas y las denunciadas confiere sustento a su validez concurrente. Es sorprendente, la estrecha similitud en el orden de prelación de los porcentajes de las transgresiones. El que no se encuentre denuncias en confección de historia clínica, ni educación médica continua, es comprensible ya que los denunciantes generalmente no poseen conocimientos suficientes para detectar errores en tales actividades, lo cual explica su exclusión en la tabla $N^{\circ} 3$. En los demás tipos de denuncias comparables, existe igual orden prelación, entre los porcentajes de transgresiones "observadas" y "denunciadas".

Como era de esperar, considerando el interés y conocimientos del tema, ningún médico de los 530 encuestados, negó la calidad de transgresión de los ítemes propuestos, lo que está a favor de la validez de contenido.

Las 6 áreas delimitadas al confeccionar la tabla $\mathrm{N}^{\circ} 2$, merecen un comentario aparte: las áreas mas frecuentes son las que afectan la relación médico-paciente, (40\%). y la relacionada con la elaboración de la historia clínica, (17\%), lo que necesariamente establece varios interrogantes: ¿algo falla en la formación de pregrado?; ¿el poderoso influjo de la tecnología de punta dentro de los exámenes auxiliares, genera disminución de la importancia de una buena anamnesis y examen clínico?. ¿Se está olvidando la tremenda importancia de la relación médico-paciente?. ¿Las transgresiones en la elaboración de la historia clínica son sólo reflejo del decremento de la importancia de la mencionada relación?, etc. 
La tercera en frecuencia 15\%), corresponde a la desactualización, más intensa en los aspectos de la ética profesional (11\%) y todo esto a pesar del internet. ¿Hemos evaluado los esfuerzos que el Colegio Médico realiza en pro de la educación médica continua? ¿Las sociedades evalúan los resultados de sus cursos? Por nuestra parte podemos adelantar que todos los eventos realizados por el CVED-CN, han sido debidamente evaluados, lo que será tema de otro reporte.

En "Área económica" son destacables las transgresiones a las normas publicitarias (5\%), y en ello habría que preguntarse si el hecho de que por ley, no es factible ningún control previo de la propaganda, haya determinado que el Código de Ética del Colegio Médico cuente para el efecto, sólo con cuatro artículos y sin establecer control previo, en lugar de los 11 del anterior que si lo establecía.

Asímismo en el "Área de relaciones sociales extra clinica", la distorsión de las relaciones entre los médicos (5\%), ya observados en trabajo anterior (14\% de las denuncias ante el Regional III Lima), es realmente preocupante. ¿Por qué los médicos nos agredimos tanto, si somos suficientemente educados y cultos?

En el "Área de transgresiones de connotación penal", aparece el aborto (4\%), tal cual se da en el trabajo sobre denuncias ¿Es el médico, en todos los casos, realmente un delincuente, o es que a veces se atreve a solucionar los problemas de mujeres angustiadas por un embarazo indeseado? El problema, aún controversial en muchos países, espera también en el nuestro una actitud más tolerante y realista que evite mayores daños y con ello sanciones injustas.

En conclusión, se ha comprobado la validez del constructo teórico, la validez concurrente así como la confiabilidad en la sexta versión del Cuestionario de Transgresiones.

Se recomienda utilizar el Cuestionario de Transgresiones, para acercarse al diagnóstico situacional ético, dentro de cualquier población de médicos, incluso a nivel nacional.

Los tipos de transgresiones más frecuentemente de tectados, pueden tomarse como contenidos priorita rios en la educación ético-médica continua.

Debe considerarse la posibilidad de incorporar conte nidos, dentro de los cursos de ética de pre y post gra do, que enfaticen las ventajas de establecer una buena relación médico paciente así como confeccionar una buena historia clínica.

Es urgente investigar, entre otros aspectos, las razones de las distorsiones en la relación interpersonal entre los médicos.

\section{Correspondencia:}

José Véliz Márquez.

Correo electrónico: jovem@upch.edu.pe

\section{REFERENCIAS BIBLIOGRAFICAS}

1. Veliz J. Características de 148 denuncias presentadas al Comité de Vigilancia Ética del Consejo Regional III CMP (1980-1999). Acta Médica Peruana 2003; 20(1): 40-47.

2. Brennan $\mathrm{T}$, Leape $\mathrm{L}$, Laird $\mathrm{N}$, et al. Incidence of adverse events and negligence in hospitalized patients: results of the Harvard Medical Practice Study I. New Engl J Med 1991; 324:370-37.

3. Garret H. Estadística en Psicología y Educación. Barcelona: Editorial Paidos Ibérica; 1983. p. 405-406.

4. Comité de Vigilancia Ética y Deontológica del Consejo Nacional. Memoria del Comité de Vigilancia Ética y Deontológica del Consejo Nacional. Diciembre del 2005

5. Véliz J. Evaluación de seminarios de ética médica en internos de medicina. Acta Médica Peruana 2003; 20(3): 117-124.

6. Véliz J. Validez y confiabilidad de un cuestionario sobre transgresiones ético-médicas. Tesis para optar el grado de magíster en bioética. Lima Perú. Universidad Nacional Mayor de San Marcos, 2004. 